\title{
Beta blockers, norepinephrine, and cancer: an epidemiological viewpoint
}

This article was published in the following Dove Press journal:

Clinical Epidemiology

28 June 2012

Number of times this article has been viewed

\section{Paul J Fitzgerald}

The Zanvyl Krieger Mind/Brain Institute, Solomon H Snyder Department of Neuroscience, Johns Hopkins University, Baltimore, MD, USA
Correspondence: Paul J Fitzgerald National Institute on Alcohol Abuse and Alcoholism (NIAAA),

5625 Fishers Lane, Room 2N09,

Bethesda, MD 20852-94I I, USA

Tel +30l 4434052

Email: pfitz@mbi.mb.jhu.edu
Abstract: There is growing evidence that the neurotransmitter norepinephrine (NE) and its sister molecule epinephrine (EPI) (adrenaline) affect some types of cancer. Several recent epidemiological studies have shown that chronic use of beta blocking drugs (which antagonize $\mathrm{NE}$ /EPI receptors) results in lower recurrence, progression, or mortality of breast cancer and malignant melanoma. Preclinical studies have shown that manipulation of the levels or receptors of NE and EPI with drugs affects experimentally induced cancers. Psychological stress may play an etiological role in some cases of cancer (which has been shown epidemiologically), and this could be partly mediated by NE and EPI released by the sympathetic nervous system as part of the body's "fight or flight" response. A less well-appreciated phenomenon is that the genetic tone of NE/EPI may play a role in cancer. NE and EPI may affect cancer by interacting with molecular pathways already implicated in abnormal cellular replication, such as the P38/MAPK pathway, or via oxidative stress. NE/EPI-based drugs other than beta blockers also may prevent or treat various types of cancer, as may cholinesterase inhibitors that inhibit the sympathetic nervous system, which could be tested epidemiologically.

Keywords: clonidine, guanfacine, aspirin, acetylcholine, epinephrine, adrenaline, sympathetic nervous system, parasympathetic nervous system, inflammation

\section{Clinical and preclinical findings}

Within the last two years, a number of retrospective epidemiological studies have found that chronic use of beta-blocking drugs is associated with lower recurrence and mortality of breast cancer, ${ }^{1,2}$ or reduced progression and mortality of breast cancer ${ }^{3,4}$ and malignant melanoma., ${ }^{5,6}$ Three earlier studies also suggested beneficial effects from prolonged use of beta blockers on general cancer risk $^{7}$ or on prostate cancer risk. ${ }^{8,9}$ Collectively, these findings are both provocative and encouraging. They are provocative because beta blockers, which have been used clinically for decades worldwide, are typically taken to treat heart-related ailments, such as arrhythmias and hypertension, not cancer. They are encouraging because beta blockers may represent a "new," and relatively safe, category of drugs for the prevention and possible treatment of a range of cancers, while also shedding light on the pathophysiological basis of some types of cancer.

A few years ago, I put forth the hypothesis that the signaling molecule norepinephrine (NE) is an etiological factor in some types of cancer. ${ }^{10,11}$ In support of this hypothesis I cited seven lines of evidence: (i) rodent studies of tumorigenesis in the context of NE manipulation, (ii) human studies of tricyclic antidepressant use and cancer rate, (iii) existence of pheochromocytoma, a cancer of the adrenal glands, (iv) cancer rates 
in families with individuals who have bipolar disorder, (v) hypertension and cancer risk, (vi) excessive body weight and cancer risk, and (vii) psychological stressors and cancer risk. These lines of evidence tend to show that increased NE release in the body, or increased numbers or sensitivity of NE receptors, is associated with increased occurrence of cancer in various organs.

Norepinephrine, and the related molecule epinephrine (EPI), also known as adrenaline, are stress hormones that activate the body's adrenoceptors, which includes the receptors that beta blockers antagonize. There are five major types of adrenoceptors in the body: beta1, beta2, beta3, alpha1, and alpha $2 ;{ }^{10}$ most beta blockers target the beta 1 and beta2 receptors. Adrenoceptors are G protein-coupled receptors that initiate second messenger signaling processes within cells that bear these receptors. Adrenoceptors are found not only in the brain, but also in nearly all, if not all, organs of the body. ${ }^{10} \mathrm{NE}$ and EPI have direct access to these organs through localized release by the sympathetic nervous system (SNS), as well as through general release into the bloodstream, as part of the body's "fight or flight" response to psychological stressors. By binding to adrenoceptors in various organs, $\mathrm{NE}$ and EPI may modulate various intracellular molecular pathways already implicated in cancer (see below).

There is a growing preclinical literature suggesting that NE and EPI may be etiological factors in cancer. For example, Sarkar et $\mathrm{al}^{12}$ have recently shown that functional balance of the sympathetic and parasympathetic branches of the autonomic nervous system may be important in rodent experimentally induced cancers, where pharmacologically increasing sympathetic NE output or blocking cholinergic parasympathetic output tended to promote cancer in their rat model. In particular, Sarkar et al ${ }^{12}$ used an innovative cellular transplantation technique that modulated beta-endorphin signaling in the hypothalamus region of the rat brain. This method may have decreased sympathetic relative to parasympathetic output, thereby reducing mammary tumor growth and metastasis. This and other studies add to data that began several decades ago, showing an effect of NE manipulation on rodent experimentally induced carcinogenesis. ${ }^{13,14}$ These data also suggest that it would be informative to epidemiologically test the effects of drugs that act on the autonomic nervous system on risk for or outcome of various types of cancer (see below).

It should be noted that some recent clinical studies have found that the use of beta blockers, as well as other NE transmission decreasing drugs, is associated with increased cancer risk and poorer survival. For example, a recent epidemiological study found that chronic beta blocker use and use of other NE decreasing drugs is associated with a slightly increased (odds ratios approximately 1.05-1.1) risk of various types of cancer combined, including colon, lung, breast, and prostate cancer. ${ }^{15}$ Another study found that longterm use ( 6 or more years) of beta blockers is associated with significantly increased risk (odds ratio 2.02) of stage IV colorectal cancer. ${ }^{16} \mathrm{~A}$ recent study of a United Kingdom database comparing cancer patients receiving beta blockers with those receiving other antihypertensive medications found slightly poorer survival rates (hazard ratio approximately 1.2) in those on beta blockers; this outcome appeared to be limited to pancreatic and prostate cancer. ${ }^{17}$ One interpretation of the data from these three studies is that persons who eventually take beta-blocking medications chronically for hypertension or other cardiac-related abnormalities have elevated endogenous (possibly genetic) NE signaling, predisposing them to various types of cancer and/or poorer cancer outcome.

In this scenario, beta-blocking drugs, at least in the manner in which they are currently used, may have a smaller effect on cancer risk or outcome than the underlying elevated NE signaling. ${ }^{18}$

In general, caution should be exercised in inferring from human drug studies that NE plays an etiological role in some cancers. Clinical epidemiological studies tend to be correlative and do not necessarily indicate that the factor in question, such as NE or a noradrenergic drug, is causative in a disease process.

Another point to consider is that different beta-blocking drugs, which vary in their specificity for different beta adrenoceptors, such as beta1 and beta2, may differentially affect cancer outcome in both preclinical and clinical settings. ${ }^{18}$ One beta blocker that has been used in a number of preclinical oncology studies, propranolol, blocks both beta1 and beta2 receptors.

\section{Psychological stress and genetics}

There is growing evidence that psychological stress is associated with increased cancer risk or poorer treatment outcome (for a recent review, see Sarkar et $\mathrm{al}^{19}$ ). For example, three recent preclinical studies have shown that stress affects experimentally induced cancers. In one study, tumor growth transiently increased after stress was brought on by housing mice singly; this process may be affected by transient changes in peripheral NE release. ${ }^{20}$ Chronic stress was found to increase ovarian tumor growth in mice, which may have been in part mediated by NE-stimulated upregulation of the cytokine interleukin- $8 .{ }^{21}$ In a mouse model of social stress, 
a number of signaling molecules, including NE and ERK protein, increased or were upregulated, and tumor progression was prevented, when the intracellular molecule cyclic adenosine monophosphate was reduced by treatment with the inhibitory neurotransmitter gamma-aminobutyric acid. ${ }^{22}$

If psychological stress affects cancer onset or progression, one possibility is that stress-related increase in the release of $\mathrm{NE}$ and EPI, and only these two molecules, is responsible for the deleterious effects on cancer risk and outcome. However, other molecules, such as neuropeptide Y (NPY) and cortisol also mediate the body's physiological stress response. Do these latter two molecules also play a role in some types of cancer? A recent study found that NPY receptors are present in the 4T1 breast cancer cell line, and that administration of NPY to these cells promoted proliferation and migration. ${ }^{23}$ In addition, it has been shown that cortisol (also known as hydrocortisone) downregulates the expression of the breast cancer susceptibility gene BRCA1 in the nonmalignant mouse mammary cell line EPH4, where such downregulation has been associated with breast cancer development. ${ }^{24}$ As we come to better understand the genetics of stress-related molecules such as NPY in humans, epidemiological investigation of the possible association between various alleles and cancer initiation or progression would be informative.

As Sarkar et $\mathrm{al}^{19}$ pointed out, the cellular and molecular mechanisms whereby NE and EPI may carry out stressrelated effects on carcinogenesis are beginning to be elucidated. They suggest that suppression of SNS functioning (via their beta-endorphin transplantation method, noted above) can result in reduced tumorigenesis via increased peripheral natural killer cell and macrophage activities, elevated levels of anti-inflammatory cytokines, and reduced levels of inflammatory cytokines, as well as changes in tumor microenvironment. In other words, NE and EPI may promote carcinogenesis through immune system dysfunction and pathological inflammation. Another point is that perhaps both the magnitude and the duration of exposure to psychological stress affect the probability of developing cancer. This is a topic that would benefit from further epidemiological retrospective investigation in persons who have been exposed to various types and duration of psychological stress.

While psychological stress, which is partly mediated by the endogenous release of NE and EPI, has already been associated with increased cancer risk in humans, ${ }^{25}$ it is also possible that genetically elevated NE/EPI tone is an etiological factor in some cases of cancer. "Tone" refers to the rather steady, baseline output of the SNS, rather than the "phasic" output of the system, where the latter is more closely associated with rapid changes in the system related to the "fight or flight" response. There appear to be genetic differences (ie, polymorphisms) in the NE component of the SNS within different persons, such as for the alpha2C receptor subtype, ${ }^{26}$ which may affect cancer risk or outcome in various individuals. Perhaps some individuals have genetically elevated NE and/or EPI transmission, including increased steady release of NE and/or increased numbers or sensitivity of adrenoceptors receiving NE input, predisposing them to cancer in a variety of organs. For example, polymorphisms of the beta 2 and beta 3 adrenoceptor genes may be associated with breast cancer risk. ${ }^{27} \mathrm{~A}$ final point on genetics is that gene $\mathrm{X}$ environment (ie, psychological stress) interactions may play an important role in some types of cancer. In this scenario, an individual with genetically elevated SNS NE tone, who is also exposed to significant psychological stress, may be especially susceptible to developing cancer, or have a worse outcome if he or she does develop cancer. The effects of NE/EPI genetics, in combination with stress and other environmental inputs, on cancer risk or outcome is a topic that could greatly benefit from epidemiological investigation.

Work by Felitti, Anda, and colleagues has suggested that psychological stress or trauma during childhood is associated with later development of cancer. ${ }^{28,29}$ In one study, they found that psychologically adverse experiences during childhood, such as physical or sexual abuse, are associated with the development of cancer in adulthood. ${ }^{28}$ More recently, they suggested that adverse childhood experiences may be associated with later development of lung cancer, even after correcting for the effects of smoking. ${ }^{29}$

\section{Etiological mechanisms}

How might elevated release of NE and EPI, or greater numbers or sensitivity of their receptors, actually cause various types of cancer? One possibility is that by binding to adrenoceptors on the outside of cells of the body's organs, they activate intracellular molecular pathways already implicated in cancer. For example, there is growing evidence that NE and adrenoceptors interact with the P38/MAPK, ${ }^{30}$ Stat $3,{ }^{31}$ and $\mathrm{PI} 3 \mathrm{~K} / \mathrm{AKT}^{32}$ signaling pathways. If so, this may provide an additional link between extracellular signaling mechanisms and intracellular signaling pathways, such as P38/MAPK, which are the focus of intensive study by molecular oncology researchers. (Epidermal growth factor is another example of an extracellular signaling molecule that affects cancer risk.) As noted above in relation to Sarkar et $\mathrm{al}^{19}$ elevated NE signaling by the SNS may have deleterious effects on cellular 
immunity and inflammatory properties. Related to this point, NE induces proliferation of rat cardiac fibroblasts, in part by increasing expression of the inflammation-modulating cytokine interleukin-6, through regulation of MAPK. ${ }^{33}$ It may be that chronically elevated NE signaling has negative effects on the immune system and inflammation, whereas acute increases in NE signaling are a healthy response to such challenges as infection, shock, or sepsis.

An additional way that NE and EPI may affect cancer is by modulating cellular oxidative damage. For example, a study on application of EPI to human leukocytes showed that it produced damaging oxygen species, induced DNA strand breakage, and such damage was antagonized by the antioxidant enzymes superoxide dismutase and catalase. ${ }^{34}$

\section{Drug studies and treatments}

Beta blockers are not the only drugs that interfere with NE and EPI signaling. Prazosin blocks the alpha 1-adrenoceptor, and it continues to be studied in relation to benign prostatic hyperplasia. ${ }^{35}$ Clonidine and guanfacine, which are alpha 2-adrenoceptor agonists used to treat hypertension, cause a general decrease in NE release, but they have barely been studied in relation to cancer, either preclinically or clinically. Clonidine has been studied to some degree in relation to pheochromocytoma; however, to my knowledge, these studies address its use as a test for the presence of this cancer, not its prevention.

It would also be worthwhile to study, preclinically and clinically, the effects of cholinesterase inhibitor drugs, such as galantamine and donepezil, which boost levels of the molecule acetylcholine, as this molecule may oppose the effects of NE in the autonomic nervous system. ${ }^{12}$ That is, acetylcholine released by the parasympathetic nervous system may oppose the effects of NE released by the SNS, thereby possibly having beneficial effects on cancer risk and outcome. It may be even more informative and therapeutic to combine beta blockers (or clonidine) with cholinesterase inhibitors, or beta blockers (or clonidine) with aspirin, ${ }^{36}$ to test for additive effects on cancer prevention and/or treatment. Holmes et $\mathrm{al}^{36}$ reported beneficial effects from long-term use of aspirin on the survival of nurses who had previously been diagnosed with breast cancer. All of the above drugs have been approved for human use for many years in the United States and elsewhere, making epidemiological studies of them feasible.

Histone deacetylase inhibitor drugs, which may be useful in treating some cancers, also may achieve this effect, by increasing cellular expression of the NE transporter, ${ }^{37}$ which could reduce the extracellular concentration of NE.
Extension of Porges' polyvagal theory might suggest that brainstem regulation of the release of acetylcholine by the tenth cranial nerve may play a role in peripheral diseases such as cancer. ${ }^{38}$

Long-term, rather than short-term, adrenoceptor-related drug treatment may be necessary to significantly reduce the probability of developing cancer, consistent with the notion that tonic, rather than phasic, release of NE and EPI may be more important in carcinogenesis, as tonic output has more of a sustained presence on cells bearing adrenoceptors in various organs. The epidemiological beta blocker studies described at the outset of this review typically measured the effects of years of exposure to these drugs, rather than hours or days.

Regarding further testing of the NE/EPI cancer hypothesis epidemiologically, it would be very informative to carry out prospective studies on the effects of the above drug treatments, ${ }^{39}$ as, to my knowledge, all of the studies published to date have been retrospective. However, additional retrospective studies also would be informative, and they are readily feasible. As we learn more about the genetics of the NE/EPI system, it would be useful to study, in more breadth and detail, the potential associations between genetic polymorphisms and various types of cancer. ${ }^{27}$

A caveat regarding the use of beta blockers as a potential means for preventing or treating cancer is that at least some of these drugs may be associated with metabolic syndrome or type 2 diabetes mellitus. ${ }^{40}$ However, vasodilating beta blockers may have beneficial or neutral metabolic effects when compared with nonvasodilating beta blockers. ${ }^{40}$ This topic would benefit greatly from further investigation, comparing costs and benefits of using these drugs for cancer prevention or treatment.

Lastly, if beta blockers, as well as other adrenoceptor-based drugs discussed above, really do represent a new and relatively safe category of drugs that may not only prevent but also treat existing cases of various types of cancer, perhaps these drugs could also be considered as a last resort intervention in terminal cases where all conventional treatments have failed. The suggestion here is that beta blockers may actually improve survival in such cases, and not merely be palliative. While I have indicated above that long-term use of beta blockers may be necessary to prevent or treat various types of cancer, perhaps these drugs, to some degree, would start working immediately to reduce further progression of the disease.

\section{Grant support}

This work was not supported by any funding source. 


\section{Disclosure}

The author has no conflicts of interest to disclose.

\section{References}

1. Powe DG, Voss MJ, Zanker KS, et al. Beta-blocker drug therapy reduces secondary cancer formation in breast cancer and improves cancer specific survival. Oncotarget. 2010;1(7):628-638.

2. Ganz PA, Habel LA, Weltzien EK, Caan BJ, Cole SW. Examining the influence of beta blockers and ACE inhibitors on the risk for breast cancer recurrence: results from the LACE cohort. Breast Cancer Res Treat. 2011;129(2):549-556.

3. Barron TI, Connolly RM, Sharp L, Bennett K, Visvanathan K. Beta blockers and breast cancer mortality: a population-based study. J Clin Oncol. 2011;29(19):2635-2644.

4. Melhem-Bertrandt A, Chavez-Macgregor M, Lei X, et al. Beta-blocker use is associated with improved relapse-free survival in patients with triple-negative breast cancer. J Clin Oncol. 2011;29(19):2645-2652.

5. Lemeshow S, Sorensen HT, Phillips G, et al. $\beta$-Blockers and survival among Danish patients with malignant melanoma: a population-based cohort study. Cancer Epidemiol Biomarkers Prev. 2011;20(10):2273-2279.

6. De Giorgi V, Grazzini M, Gandini S, et al. Treatment with $\beta$-blockers and reduced disease progression in patients with thick melanoma. Arch Intern Med. 2011;171(8):779-781.

7. Algazi M, Plu-Bureau G, Flahault A, Dondon MG, Le MG. Could treatments with beta-blockers be associated with a reduction in cancer risk? Rev Epidemiol Sante Publique. 2004;52(1):53-65. Article in French.

8. Perron L, Bairati I, Harel F, Meyer F. Antihypertensive drug use and the risk of prostate cancer (Canada). Cancer Causes Control. 2004;15(6):535-541.

9. Rodriguez C, Jacobs EJ, Deka A, et al. Use of blood-pressure-lowering medication and risk of prostate cancer in the Cancer Prevention Study II Nutrition Cohort. Cancer Causes Control. 2009;20(5):671-679.

10. Fitzgerald PJ. Is norepinephrine an etiological factor in some types of cancer? Int J Cancer. 2009;124(2):257-263.

11. Fitzgerald PJ. Testing whether drugs that weaken norepinephrine signaling prevent or treat various types of cancer. Clin Epidemiol. 2010;2:1-3.

12. Sarkar DK, Zhang C, Murugan S, et al. Transplantation of $\beta$-endorphin neurons into the hypothalamus promotes immune function and restricts the growth and metastasis of mammary carcinoma. Cancer Res. 2011;71(19):6282-6291.

13. Gurkalo VK, Zabezhinskii MA, Pliss GB, Krylov SS. Adrenergic component in the hepatotropic, carcinogenic effect of diethylnitrosamine. Biull Eksp Biol Med. 1977;83(4):455-458. Article in Russian.

14. Tatsuta M, Iishi H, Baba M, Nakaizumi A, Ichii M, Taniguchi H. Promotion by bombesin of gastric carcinogenesis induced by $\mathrm{N}$-methyl-N'-nitro-N-nitrosoguanidine in Wistar rats. Cancer Res. 1989;49(19):5254-5257.

15. Friedman GD, Udaltsova N, Habel LA. Norepinephrine antagonists and cancer risk. Int J Cancer. 2011;128(3):737-738.

16. Jansen L, Below J, Chang-Claude J, Brenner H, Hoffmeister M. Beta blocker use and colorectal cancer risk: population-based case-control study. Cancer. May 14, 2012. doi:10.1002/cncr.26727. [Epub ahead of print.]

17. Shah SM, Carey IM, Owen CG, Harris T, Dewilde S, Cook DG. Does $\beta$-adrenoceptor blocker therapy improve cancer survival? Findings from a population-based retrospective cohort study. Br J Clin Pharmacol. 2011:72(1):157-161

18. Fitzgerald PJ. Author's reply to: Norepinephrine antagonists and cancer risk. Int J Cancer. 2011;128:738-739.

19. Sarkar DK, Murugan S, Zhang C, Boyadjieva N. Regulation of cancer progression by $\beta$-endorphin neuron. Cancer Res. 2012;72(4): 836-840.
20. Madden KS, Szpunar MJ, Brown EB. Early impact of social isolation and breast tumor progression in mice. Brain Behav Immun. May 17, 2012. [Epub ahead of print.]

21. Shahzad MM, Arevalo JM, Armaiz-Pena GN, et al. Stress effects on FosB- and interleukin-8 (IL8)-driven ovarian cancer growth and metastasis. J Biol Chem. 2010;285(46):35462-35470.

22. Schuller HM, Al-Wadei HA, Ullah MF, Plummer HK 3rd. Regulation of pancreatic cancer by neuropsychological stress responses: a novel target for intervention. Carcinogenesis. 2012;33(1):191-196.

23. Medeiros PJ, Al-Khazraji BK, Novielli NM, Postovit LM, Chambers AF, Jackson DN. Neuropeptide Y stimulates proliferation and migration in the 4T1 breast cancer cell line. Int J Cancer. 2012;131(2):276-286.

24. Antonova L, Mueller CR. Hydrocortisone down-regulates the tumor suppressor gene BRCA1 in mammary cells: a possible molecular link between stress and breast cancer. Genes Chromosomes Cancer. 2008;47(4):341-352.

25. Metcalfe C, Davey Smith G, Macleod J, Hart C. The role of self-reported stress in the development of breast cancer and prostate cancer: a prospective cohort study of employed males and females with 30 years of follow-up. Eur J Cancer. 2007;43(6):1060-1065.

26. Neumeister A, Charney DS, Belfer I, et al. Sympathoneural and adrenomedullary functional effects of alpha2C-adrenoreceptor gene polymorphism in healthy humans. Pharmacogenet Genomics. 2005;15(3):143-149.

27. Huang XE, Hamajima N, Saito T, et al. Possible association of beta2and beta3-adrenergic receptor gene polymorphisms with susceptibility to breast cancer. Breast Cancer Res. 2001;3(4):264-269.

28. Felitti VJ, Anda RF, Nordenberg D, et al. Relationship of childhood abuse and household dysfunction to many of the leading causes of death in adults. The Adverse Childhood Experiences (ACE) Study. Am J Prev Med. 1998;14(4):245-258.

29. Brown DW, Anda RF, Felitti VJ, et al. Adverse childhood experiences are associated with the risk of lung cancer: a prospective cohort study. BMC Public Health. 2010;10:20.

30. Huang XY, Wang HC, Yuan Z, Huang J, Zheng Q. Norepinephrine stimulates pancreatic cancer cell proliferation, migration and invasion via $\beta$-adrenergic receptor-dependent activation of P38/MAPK pathway. Hepatogastroenterology. 2012;59(115):889-893.

31. Landen CN Jr, Lin YG, Armaiz Pena GN, et al. Neuroendocrine modulation of signal transducer and activator of transcription-3 in ovarian cancer. Cancer Res. 2007;67(21):10389-10396.

32. Yanagawa Y, Matsumoto M, Togashi H. Enhanced dendritic cell antigen uptake via alpha2 adrenoceptor-mediated PI3K activation following brief exposure to noradrenaline. J Immunol. 2010;185(10):5762-5768.

33. Leicht M, Briest W, Zimmer HG. Regulation of norepinephrine-induced proliferation in cardiac fibroblasts by interleukin- 6 and p42/p44 mitogen activated protein kinase. Mol Cell Biochem. 2003;243(1-2):65-72.

34. Crespo ME, Bicho MP. Membrane-mediated effects of catecholamines on the DNA of human leukocytes: the role of reactive oxygen species. Biol Signals. 1995;4(2):78-85.

35. Liao CH, Guh JH, Chueh SC, Yu HJ. Anti-angiogenic effects and mechanism of prazosin. Prostate. 2011;71(9):976-984.

36. Holmes MD, Chen WY, Li L, Hertzmark E, Spiegelman D, Hankinson SE. Aspirin intake and survival after breast cancer. J Clin Oncol. 2010; 28(9):1467-1472.

37. More SS, Itsara M, Yang X, et al. Vorinostat increases expression of functional norepinephrine transporter in neuroblastoma in vitro and in vivo model systems. Clin Cancer Res. 2011;17(8):2339-2349.

38. Porges SW. Orienting in a defensive world: mammalian modifications of our evolutionary heritage. A Polyvagal theory. Psychophysiology. 1995;32(4):301-318.

39. Pasquier E, Ciccolini J, Carre M, et al. Propranolol potentiates the anti-angiogenic effects and anti-tumor efficacy of chemotherapy agents: implication in breast cancer treatment. Oncotarget. 2011; 2(10):797-809.

40. Taylor AA, Bakris GL. The role of vasodilating beta-blockers in patients with hypertension and the cardiometabolic syndrome. Am J Med. 2010;123(7 Suppl 1):S21-S26. 


\section{Publish your work in this journal}

Clinical Epidemiology is an international, peer-reviewed, open access journal focusing on disease and drug epidemiology, identification of risk factors and screening procedures to develop optimal preventative initiatives and programs. Specific topics include: diagnosis, prognosis, treatment, screening, prevention, risk factor modification, systematic

Submit your manuscript here: http://www.dovepress.com/clinical-epidemiology-journal reviews, risk \& safety of medical interventions, epidemiology \& biostatical methods, evaluation of guidelines, translational medicine, health policies \& economic evaluations. The manuscript management system is completely online and includes a very quick and fair peer-review system, which is all easy to use. 